\title{
A Case Study on the Integration of GPS Concepts in a PLM Based Industrial Context
}

\author{
Francesco Ricci ${ }^{1}$, Joel Sauza Bedolla ${ }^{1}$, Javier Martinez Gomez ${ }^{1,2}$, \\ Suela Ruffa ${ }^{1}$, Manuela De Maddis ${ }^{1}$, and Paolo Chiabert ${ }^{1}$ \\ ${ }^{1}$ Politecnico di Torino, C.so Duca degli Abruzzi 24, Torino 10129, Italy \\ ${ }^{2}$ Universidad Industrial de Santander, Calle 9 Cra.27, Bucaramanga, Colombia
}

\begin{abstract}
The innovative principles of the Geometrical Product Specification and Verification "GPS" framework promise a breakthrough in the management of geometry-related information and will lead to effective product management based on the concept of uncertainty minimization. GPS standards define a technical language completely based on mathematics to achieve these objectives and, for this reason, only major companies are able to cope with it. This paper presents a new methodological and software tool developed for integrating GPS principles into Product Lifecycle Management (PLM). It captures and structures the information gravitating around product geometry and finally encapsulates GPS principles into a user-friendly platform suitable for enterprises of any scale. This work also represents the first complete application of GPS standards to a product lifecycle, which is useful for training in the field.
\end{abstract}

\section{Introduction}

The work of the ISO Technical Committee 213 (ISO/TC-213) on Geometrical Product Specification and Verification (GPS) started in 1992 to modernize and improve on more than fifty years of industrial drawing and tolerancing practice consolidated in GD\&T (Geometric Dimensioning and Tolerancing) standards [1]. The aim of TC-213 is to provide tools (the GPS technical language) for the economic management of variability in products and processes. GPS maintains the basic symbols of GD\&T and introduces an operation-based representation of geometrical specification and verification procedures. It then uses the concept of uncertainty to quantify the system efficiency and identify the process areas on which to focus investments or reduce costs.

Since the GPS language is completely based on mathematics, the user's first impression is usually that it is more geared to academics than to industry. Hence, GPS principles need to be encapsulated into user-friendly applications that can be integrated into companies' Product Data Management (PDM) systems. GPS ensures the unambiguous declaration of the products geometrical requirements. However, the way this information is created, modified and exchanged is not within its declared bounds.

Nevertheless the GPS approach prepares the ground for a Product Lifecycle Management (PLM) system to assess and minimize the uncertainty generated at different steps of a product lifecycle. PLM is a strategic business approach that integrates all 
the information related to the company's products and activities, throughout the different phases of a product lifecycle, and allows its sharing within and between organizations [2,3]. Its aim is to ensure the fast, easy and trouble-free finding, refining, distribution and reutilization of the data required for daily operations [4].

Nowadays, GPS language is still in a state of dynamic change and continuous improvement [5]. While the main framework has already been drawn, innovative principles are still being studied by ISO experts, academics and industry [6]. At the same time, work is being done to integrate these new principles in the PDM practices. This paper is concerned both with tools, to deliver softwares able to handle the new kind of geometrical information, and with training, to spread the concepts on which GPS relies. This paper briefly presents the results that the Great $2020^{1}$ research project achieved on this topic. An experimental case study is used to explore all the process areas involved in GPS (see Fig. 1.a) with particular attention to the implementation of uncertainty-based product management. In particular, a flatness requirement (the datum feature A of the workpiece shown in Fig. 1.b) is considered, realizing the first full application of a set of GPS standards (technically called "chain of standards" [6]).

\section{The Novelty of GPS Standards}

The GPS project was born to enhance the GD\&T language, preserving the semantics of geometrical tolerances while adding more prescriptions aimed at guiding the verification procedures. These prescriptions are not provided aside the tolerance cartouche, but become part of the tolerance semantics: they are embedded in it by means of a detailed operation-based description, which sets clear limits for interpretation and becomes a guideline for proper verification [7]. However, even if specifications have all the attributes to fully prescribe the verification strategy, in the GPS fundaments there
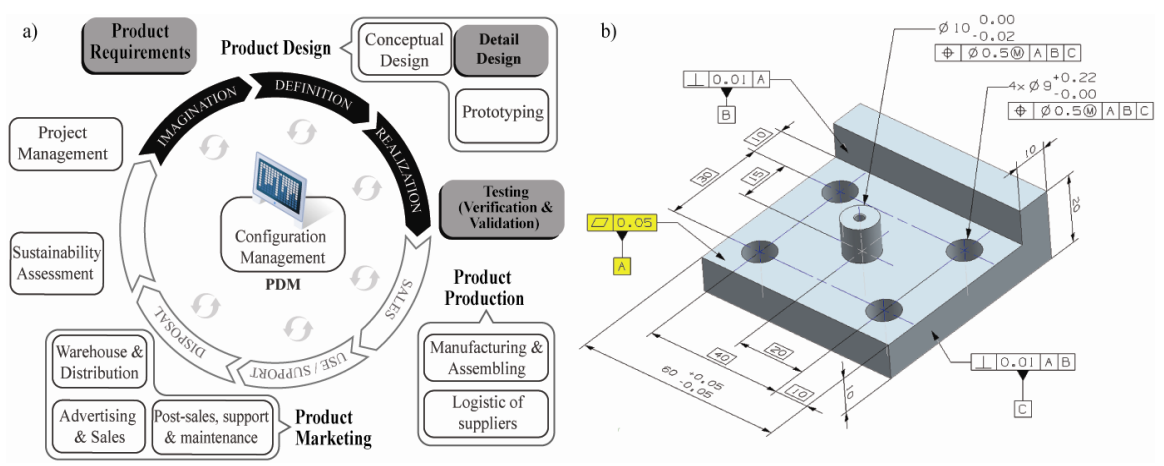

Fig. 1. a) Highlight of the stages of product lifecycle (in black) and process areas that are interested by the GPS standards (in gray) and b) the case study used to explore them

\footnotetext{
${ }^{1}$ The GREAT 2020 research project, funded by the Piedmont Region, involved the Polytechnic of Turin and several large, medium, and small Italian enterprises operating in the aerospace and aeronautic field, both as designers and manufacturers.
} 
is the awareness that some uncertainty arises anytime the product information is exchanged between two parties or when it comes to cope with the limitations of real measuring instruments.

The GPS language looks at products on a perspective that is broader than that of GD\&T, going further the mere definition of geometrical specifications and compliance verification [8]. The final aim of a workpiece is to perform a function (on its own or in the assembly of a more complex machine), therefore a proper assessment of its quality has to consider the consistency of the actual workpiece geometry with the functionality it is designed and demanded to satisfy. Though it may seem to be a nuance, this is a breakthrough point with respect to GD\&T.

GPS gives birth to a series of uncertainty contributions that join the consolidated concept of measurement uncertainty [9] (innovatively divided into the components of method uncertainty and implementation uncertainty) in order to consider also the completeness and unambiguity of specifications (specification uncertainty), the capability to state the compliance of geometry with respect to the geometrical specifications (compliance uncertainty) and the adequacy of the geometrical specification to guarantee the functional needs (correlation uncertainty) [10]. All these uncertainty contributions participate in the total uncertainty, which describes the adequacy of the actual (measured) feature to guarantee the intended workpiece functionality, according to the scheme presented in Fig. 2.

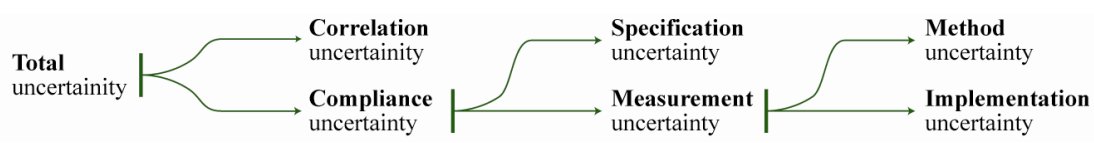

Fig. 2. Composition scheme for the GPS uncertainty contributions

The different terms of uncertainty introduced by GPS are powerful estimators of the quality of each stage of product development, starting from the first phase of design until the verification prior to delivery. Hence, if they are quantitatively estimated, they can become the currency for an effective product management [6]. E.g. a high specification uncertainty means that more efforts should be concentrated on the design phase while a too high measurement uncertainty underlines a verification process that is too poor for the job purpose.

The remaining part of this section uses the flatness case study to present the differences of specification and verification with GD\&T and GPS standards, explaining the meaning and usefulness of each term of uncertainty.

\section{Specification and Verification at the Time of GD\&T}

GD\&T standards, born in 1966 [11], had been shaped to cope with the requirements of an industry led by the automotive and aeronautic/aerospace fields, hence, with the need to guarantee interchangeability within increasingly larger assemblies in a context strongly characterized by mass production. A set of symbols, the geometrical tolerances, was designed to control the geometry of components and guarantee the functional requirements of single workpieces as well as the assembly requirements (design for assembly). The semantic of these symbols is very clear and soundly defined from a geometrical and mathematical point of view (see the example of a flatness specification depicted in Fig. 3.a). However, it can be quite cumbersome when dealing with verification issues. 
While the GD\&T standards were defined, the economy of mass production, and the relative simplicity of most of specifications, allowed the realization of functional gauges that easily reproduced the envelope principle and the verification of mating conditions [12]. GD\&T was born in symbiosis with these tools, and few recommendations were sufficient to guide most of the verification processes [13]. Particularly, the verification strategy for a flatness specification would require a measurement setup like the one shown in Fig. 3.b. Here the flatness error, whose values should be lower than the flatness tolerance for the feature to be acceptable, is represented by the maximum travel registered by the dial gauge.

a)

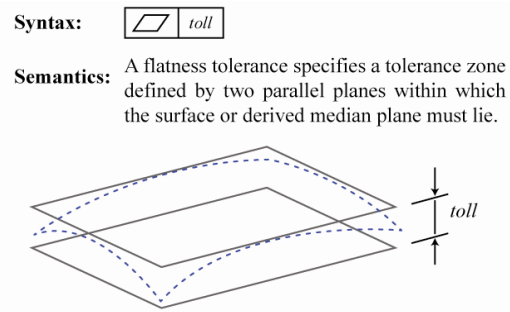

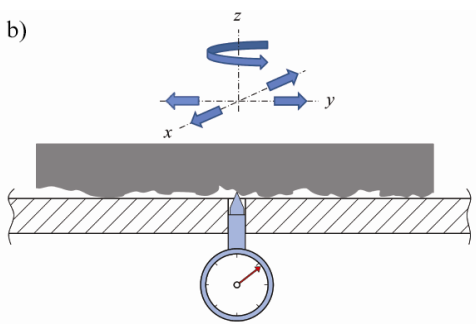

Fig. 3. a) Syntax and semantics of a flatness specification and b) measurement setup suggested by the GD\&T standards for its verification

Nowadays the shift towards mass customization, the increased complexity of components, and the demand for precision features constrain the evolution of measuring equipments towards greater flexibility. The best mix of flexibility and accuracy, in this field, is represented by Coordinate Measuring Machines (CMM); instruments that have literally revolutionized the way of thinking about and taking measurements [14]. The characteristics of this revolution, and the aids provided by the GPS language to cope with the issues of the new measurement philosophy, are analyzed thoroughly in the next section.

\section{Specification and Verification Based on GPS}

The GPS language is based on seven operations that can be combined in operators to define geometrical specifications and verification procedures. According to the duality principle [11], these operations are defined by the designer on the skin model (a mental representation that is used to imagine the deviations from the nominal geometry that could be introduced by manufacturing processes), registered in the tolerance callout, and then replicated by the metrologist during verification procedures on the real workpiece. A graphical example of the operations necessary for defining and verifying a flatness specification (tolerance) is given in Fig. 4. Particularly each operation is completely addressed by the tolerance callout and consists of:

1) Partition: isolation of the feature to which the specification refers to.

2) Extraction: acquisition of the information necessary to define the feature characteristics. In the case of Fig. 4, it is a measurement where the distance between sampling points is minor than $0.357 \mathrm{~mm}$ in order to comply with the filter cut-off wavelength [15]). 
3) Filtration: elaboration of measurement results in order to separate the content of deviation to which the specification refers to. Only the error components with a wavelength greater than $2.5 \mathrm{~mm}$ are to be considered for the assessment of the flatness deviation.

4) Association: a nominal flatness feature is fitted to the filtered measurement points according to the specified association criterion (Minimum Zone).

5) Evaluation: operation that returns the value of flatness deviation as the maximum distance of the filtered measurement points from the associated nominal feature.

After the verification operator has been implemented, the compliance with specifications can be assessed by comparing the results of the evaluation operation against the geometrical specification, according to the default rule provided by ISO [16] or to different agreements between customer and supplier.

As Fig. 5 shows, specification operator is defined complete if the GPS tolerance callout contains all the information necessary to completely define the verification procedure (regardless the measuring instrument). Verification operations are labeled perfect if they comply with the specification operator (even though they are unavoidably affected by implementation uncertainty, when they come to be implemented with a real measuring instrument) and simplified if they intentionally introduce some deviation (deviations add method uncertainty). If the specification is not complete, as for a GD\&T geometric tolerance, every choice for the verification operations implies specification uncertainty as, for example, a metrologist that is using a CMM do not exactly know how many points to sample or the association criterion he should use to analyze them. With some examples presented here we can improve the understanding of verification operators and GPS uncertainties, however we recommend the ISO/TS 17450-2 [10] for a thorough reading.

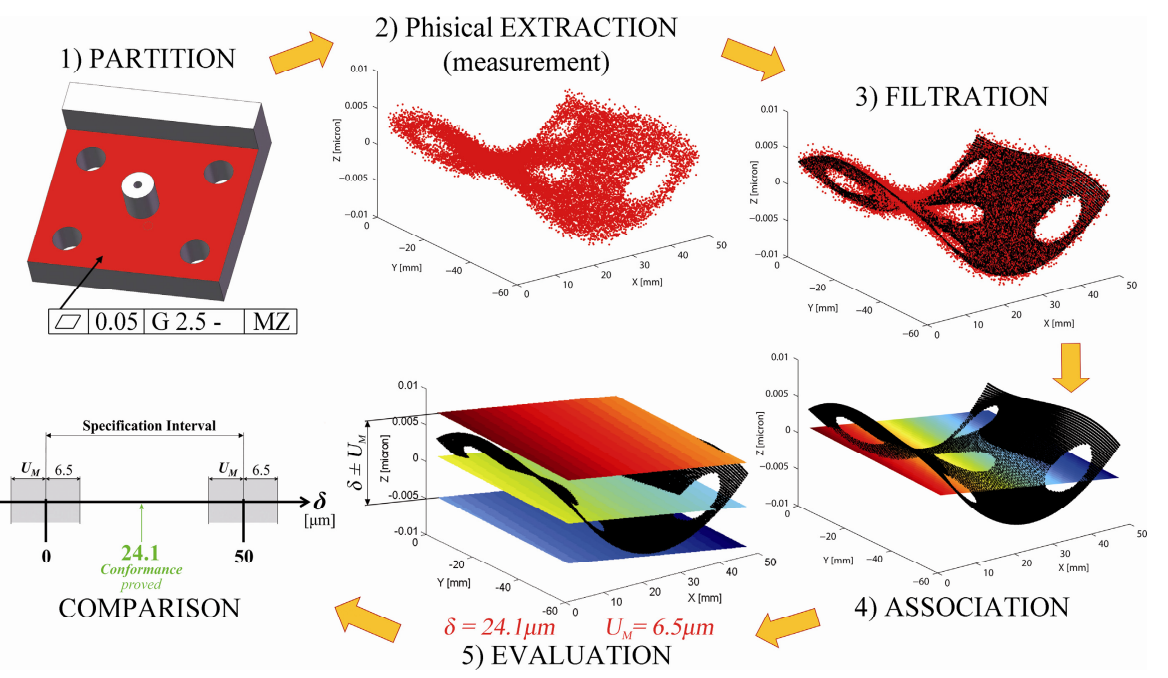

Fig. 4. Operations that define a verification operator fully compliant with specifications 


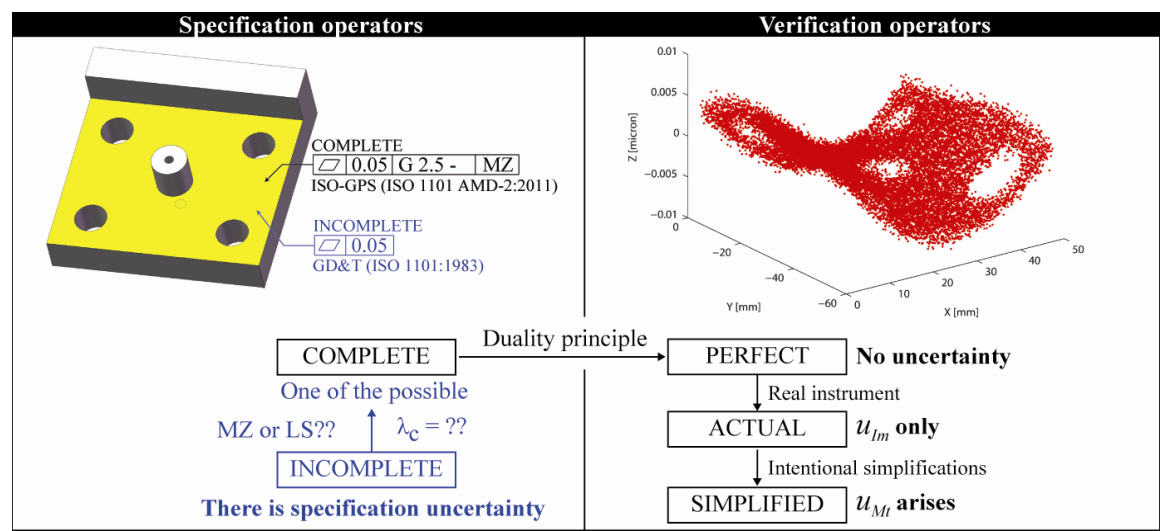

Fig. 5. GPS specification and verification operators with uncertainty contributions

For example, Fig. 6 shows the effect of association criteria on the assessment of flatness error. Two different criteria can be used according to GPS standards: Least Squares (LS) and Minimum Zone (MZ). The former always estimates an error larger than the latter, in this particular case $5 \mu \mathrm{m}$ larger. Hence, if the LS method is used instead of the required MZ, the $5 \mu \mathrm{m}$ difference shall be accounted as method uncertainty. On the other hand, if the specification is incomplete and does not explicitly require a particular association criterion, the same $5 \mu \mathrm{m}$ difference shall be accounted as specification uncertainty whichever criterion is used.

The number of sampling points used to inspect the measurand, and their spatial distribution on the surface, can be a source of uncertainty too. If the reduction of sampling points is intentional, its effect should be accounted as method uncertainty, otherwise (e.g. the cutoff wavelength is not indicated in the tolerance callout as the specification is not complete) it should be accounted as specification uncertainty.
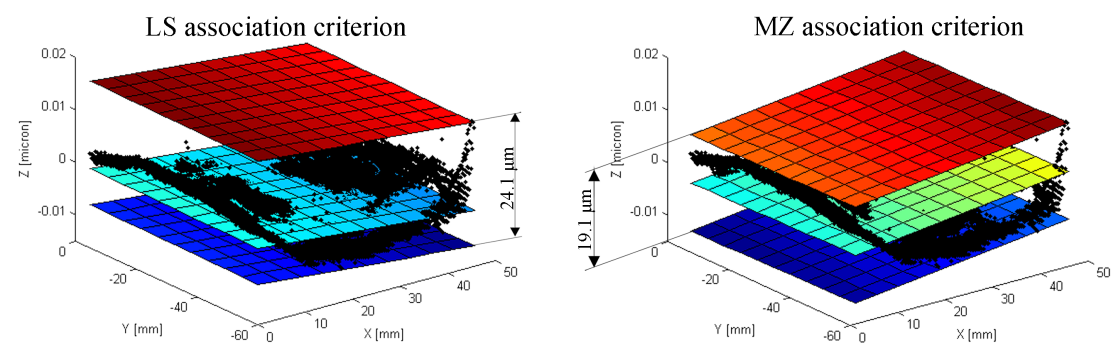

Fig. 6. Analysis of a cloud of measurement points with the LS and MZ association criteria. LS overestimates the flatness error of $5 \mu \mathrm{m}$. 


\section{Achieving a GPS Based PLM}

\section{Managing GPS Data}

In order to integrate all GPS-related product information and allow budgeting on the basis of uncertainty the authors developed a data model based on Category Theory [17]. The data model suites the main scenarios that can occur in verification of products geometry: 1) serial inspection of mass productions, 2) verification of different workpieces in small numbers. Two different strategies were designed for estimating the compliance uncertainty in each scenario. Respectively, the strategy for scenario 1 maximizes the reuse of manufacturing process information, while the one for scenario 2 is based on the structuring, storage and retrieval of measurement experience on features with a similar geometry. In both scenarios the data model enables designers and metrologists to predict (not only to assess) the uncertainties and costs associated with a given specification plus verification strategy; thus to use it for design purposes too. For a thorough presentation, reader refers to [18]. The data model was hence implemented into an object oriented programming language to obtain the VerificationManager software demonstrator. Until now it handles flatness features only, but can be expanded to the specification and verification of other geometrical features thanks to its general approach.

\section{Integrating GPS into PLM}

One of the main aims of the research project has been achieved by renewing the PLM system of each company involved, orienting it toward the GPS philosophy in order to allow the integration of instruments such as the VerificationManager. Amongst the different methodological approaches and modeling languages used for understanding and representing complex organization systems, visual representation was chosen for its capacity to show what lies within, to clarify relations, answer questions, and understand things that cannot be captured so readily in other forms [17]. We therefore developed a formal Visualization Model (VM) of enterprise processes that offers a graphic representation of the main elements of a product lifecycle (processes, people, tools, and information) involved in GPS and makes it possible to address them on an overall level [18].

Fig. 7 shows how the VM works. Particularly, starting from the workflow of GPSrelated design and verification activities, that is shown in Fig. 7.a, a Decomposition Diagram (DD) is prepared for each activity. As an example, the DD shown in Fig. 7.b breaks down the "Measurement planning" into 5 simpler operations. Besides collecting simpler operations, DDs define the skills and tools that the metrologist shall use, the input items he will receive and the expected output documents. Notice that, at this level, the metrologist is not a person but a role that can be interpreted by a single person rather than a team. Fig. 7.c shows an example of output document obtained with the VerificationManager: a forecast of the uncertainties and cost of the verification strategy the metrologist is planning. A similar graphical representation, which for the sake of brevity is not reported here, describes each activity of the workflow. 


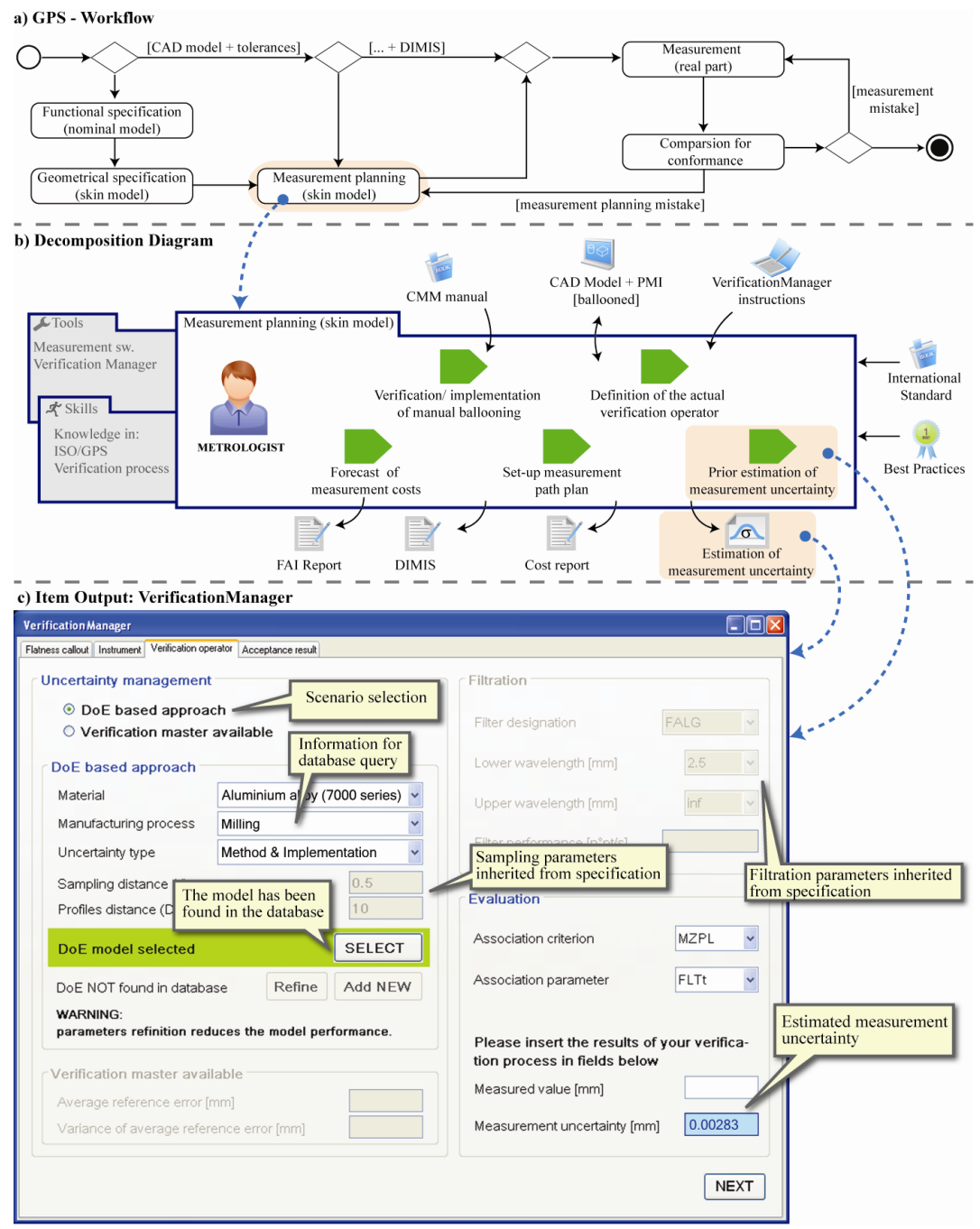

Fig. 7. VM of a GPS-based PLM: a) Workflow of design and verification activities; b) DD of one of the workflow activities: "Measurement planning"; c) Example of output item: "Estimation of the measurement uncertainty" using the VerificationManager tool

From the point of view of software implementation, at the state of the art, the VerificationManager is just a demonstrator and needs to be manually fed by users to work. Basically it needs to collect information about the complete specification operator and the actual verification operator (considering also the environment, the measurement strategy, the instrument at hand, etc.) to correctly assess the scenario, within those resumed in Fig. 5, and retrieve and elaborate the proper data for estimating the compliance uncertainty and the associated costs. 
In a scenario of greater maturity of GPS, with the possibility to define complete specification operators within CAD environments, the approach proposed by the VerificationManager should be embedded in PLM systems. The main advantages would be from the end-user point of view (many parameters would be available in the system and no manual feed would be required) and particularly from the system point of view. It provides a framework for structuring, retrieving and reusing, hence adding value to, the experience gained by companies.

\section{Conclusions}

The case study presented in this paper is the first full application, in literature, of a GPS chain of standards in PLM. The proposed approach, which integrates GPS into PLM systems, reaches every level of extended enterprise. Complete specifications can be transmitted from the design phase to manufacturing and the verification of geometrical compliance. The uncertainty introduced at each step is tracked carefully and used as a decision support tool (it is translated into a cost and used for budgeting) to improve the definition of geometrical specifications and the design of verification strategies. This work leads the way for an effective and widespread use of GPS concepts in manufacturing companies, providing a simple hands-on instrument for PLM training and implementation. While the Visualization Model is general enough to be used by any company, the GPS encapsulation needs to be extended from the flatness case study to the whole range of geometric features.

Acknowledgments. This work has been carried out with the founding of the Piedmont Region (Italy) in the framework of the GREAT 2020 research project.

\section{References}

1. Bennich, P.: Chains of Standards - A new Concept in GPS Standards. The American Society of Mechanical Engineers 7(1), 29-38 (1994)

2. Sudarsan, R., Fenves, S.J., Sriram, R.D., Wang, F.: A product information modeling framework for product lifecycle management. Computer-Aided Design 37(13), 1399-1411 (2005), doi:10.1016/j.cad.2005.02.010

3. CIMdata PLM Market Trends \& Evolution. CIMdata, Paris, France (2009)

4. Saaksvuori, A., Immonen, A.: Product Lifecycle Management, 3rd edn. Springer (2008)

5. Humienny, Z.: State of art in standardization in GPS area. CIRP Journal of Manufacturing Science and Technology 2(1), 1-7 (2009), doi:10.1016/j.cirpj.2009.06.007

6. ISO, Geometrical product specifications (GPS) - Masterplan. ISO/TR 14638. ISO, Geneve (1995)

7. ISO, Geometrical product specifications (GPS) - General concepts - Part 1: Model for geometrical specification and verification. ISO/TS 17450-1. ISO, Geneve (2005)

8. Srinivasan, V.: An Integrated View of Geometrical Product Specification and Verification. In: 7th CIRP Seminar on Computer-Aided Tolerancing, April 24 (2001)

9. ISO/IEC, Guide to the Expression of Uncertainty in Measurement (GUM: 1995). Guide 98-3. BIPM, IEC, IFCC, ISO, IUPAP, IUPAC, OIML (2008) 
10. ISO, Geometrical product specifications (GPS) - General concepts - Part 2: Basic tenets, specifications, operators and Uncertainties. ISO/TS 17450-2. ISO, Geneve (2002)

11. ISO, Geometrical product specification (GPS) - Fundamentals - Concepts, principles and rules ISO 8015. ISO, Geneve (2011)

12. Chiabert, P., Lombardi, F., Orlando, M.: Benefits of geometric dimensioning and tolerancing. Journal of Materials Processing Technology 78(1-3), 29-35 (1998), doi:10.1016/s0924-0136(97)00459-7

13. ISO, ISO system of limits and fits - Part 2: Inspection of plain workpieces. ISO/R 1938. ISO, Geneve (1971)

14. Weckenmann, A., Knauer, M., Kunzmann, H.: The Influence of Measurement Strategy on the Uncertainty of CMM-Measurements. Annals of the CIRP 47(1), 451-454 (1998)

15. ISO, Geometrical product specifications (GPS) - Straightness - Part 2: Specification operators. ISO/TS 12780-2. ISO, Geneve (2003)

16. ISO, Geometrical product specifications (GPS) - Inspection by measurement of workpieces and measuring equipment - Part 1: Decision rules for proving conformance or nonconformance with specifications. ISO 14253-1. ISO, Geneve (1999)

17. Friendly, M.: Milestones in the history of thematic cartography, statistical graphics, and data visualization. Engineering 9(2) (2009)

18. Chiabert, P., Lombardi, F., Sauza Bedolla, J., Martinez Gomez, J.: Visualization Model for Product Lifecycle Management Annals of Faculty Engineering Hunedoara, vol. 11 (1) (2013) 